\title{
Gibt es einen neuen, langsam wachsenden Melanom-Typ?
}

Die Inzidenz des kutanen Melanoms hat in den vergangenen Jahrzehnten in den Industrieländern dramatisch zugenommen. Diesen Trend sieht man allerdings nicht, was Mortalität oder das Vorkommen dicker Melanome angeht. Möglicherweise, so wird spekuliert, reflektiert die höhere Inzidenz dünner Melanome eine bessere Prävention der Neoplasie: Im Resultat würden vermehrt dünne und besonders langsam wachsende Melanome gefunden.

n einer multizentrischen retrospektiven Studie wollte ein internationales Forscherteam prüfen, ob und wie gut diese Annahme durch Befunde gestützt

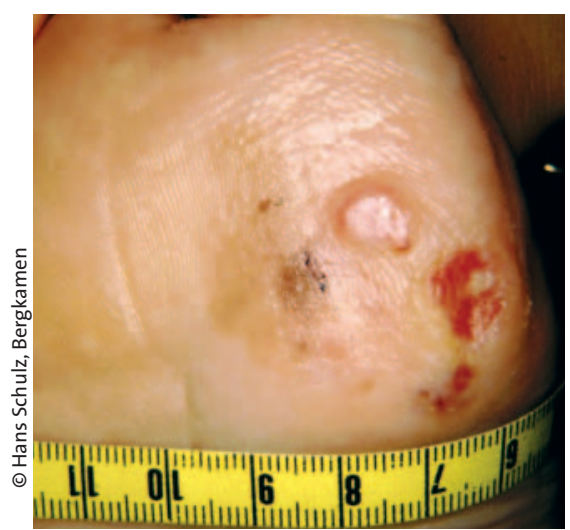

Leider nicht langsam wachsendes malignes akrolentiginöses Melanom der Fußsohle bei einer 65 -jährigen Patientin. wird. Aus einer 10.000 Melanome umfassenden Datenbank von 15 Zentren in Europa, USA und Australien wählte man solche Tumoren aus, die erst nach einer Beobachtungszeit von einem Jahr oder mehr exzidiert wurden - meist, weil sie zu Beginn so unauffällig waren. Zur Verfügung standen Daten von 103 solcher Melanome, die alle längere Zeit (zwischen 12 und 120 Monaten) beobachtet wurden, bevor man sich zur Operation entschloss. Von diesen wurden die klinisch-dermoskopischen Merkmale und Wachstumsmuster bei der ersten Konsultation und der letzten Follow-up-Untersuchung analysiert.

Nach einer medianen Beobachtungszeit von 20 Monaten befanden sich die meisten Läsionen noch in situ oder waren früh-invasiv (mediane Breslow-Dicke: $0,48 \mathrm{~mm}$ ). Nur drei der 103 untersuchten
Melanome wiesen eine Tumordicke von $1 \mathrm{~mm}$ oder mehr auf.

Die häufigsten initialen Charakteristika waren asymmetrische Pigmentierung, retikuläres Muster und regressive Veränderungen. Die Mehrzahl der Neoplasien veränderte sich während der Beobachtungszeit nur geringfügig, mit unter $2 \mathrm{~mm}$ Größenzunahme. Deutlichere Veränderungen, definiert als asymmetrische Strukturveränderung plus Wachstum von $2 \mathrm{~mm}$ oder Auftreten neuer Melanom-spezifischer Kriterien, fanden sich erst nach im Median 33 Monaten.

Fazit: Die Ergebnisse unterstützen das Postulat, dass es eine (neue) Subgruppe sehr langsam wachsender Melanome gibt. Speziell bei Patienten mit multiplen Nävi dürfte sich die Identifizierung solcher Hautveränderungen schwierig gestalten. Die Autoren halten hier eine längere Beobachtung verdächtiger Läsionen für nötig: Auf der einen Seite sollen auch Veränderungen erfasst werden, die erst nach einem oder zwei Jahre auftreten, auf der anderen Seite gilt es, unnötige Exzisionen benigner Veränderungen zu vermeiden.

wpa

Argenziano $G$ et al. Slow-growing melanoma: a dermoscopy follow-up study. Br J Dermatol. 2010; 162: 267-73.

\section{Neue Ursache für Uhrglasnägel}

_ Der 43-jährige Mann erlitt eine lazerierende Verletzung im Bereich des linken Handgelenks mit Verletzung des Nervus medianus und der Sehne des Musculus flexor carpi ulnaris. Postoperativ fiel bei dem Patienten auf, dass sich im Versorgungsgebiet des linken Nervus medianus am Daumen, Zeigefinger und Mittelfinger Uhrglasnägel ausbildeten.

Trommelschlägelfinger und Uhrglasnägel sind bei Erkrankungen des kardiorespiratorischen und gastrointestinalen Systems gut bekannt, wobei sie allerdings beidseitig auftreten. Das einseitige Auftreten im Bereich des Versorgungsgebiets eines lädierten Nerven weist auf

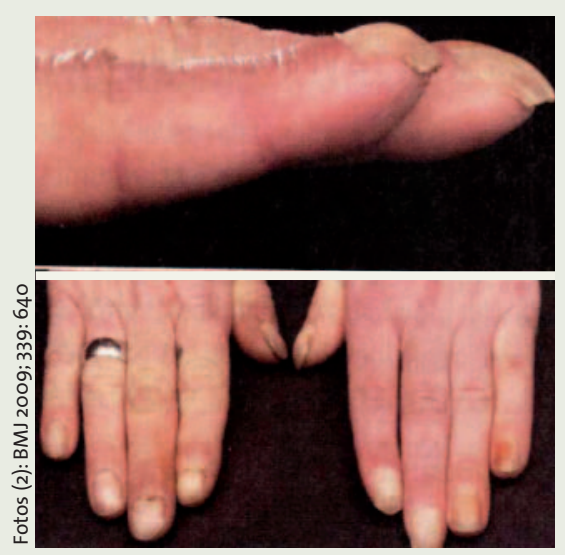

Uhrglasnägel nach Verletzung des Nervus medianus. die Pathogenese dieses Phänomens hin, da es im Rahmen eines gestörten neurozirkulatorischen Reflexes zur Ausbildung eines verstärkten Blutflusses über multiple arteriovenöse Shunts in den distalen Phalangen kommt. Dies führt zu einer Hypertrophie und Hyperplasie des Weichteilgewebes. Ähnliche Mechanismen spielen bei Verletzungen des Plexus brachialis, bei der Schultersubluxation, beim Pancoasttumor, bei der Sarkoidose und bei der Herpesinfektion im Bereich der Finger eine Rolle.

H. S. FüeßI

O'Halloran D, Webb J Brit Med J 2009; 339: 640. 